\title{
On Computation Degree-Based Topological Descriptors for Planar Octahedron Networks
}

\author{
Wang Zhen, ${ }^{1}$ Parvez Ali, ${ }^{2}$ Haidar Ali $\mathbb{D},{ }^{3}$ Ghulam Dustigeer, ${ }^{4}$ and Jia-Bao Liu $\mathbb{D}^{5}$ \\ ${ }^{1}$ School of Computer Engineering, Anhui Wonder University of Information Engineering, Hefei 231201, China \\ ${ }^{2}$ Department of Mechanical Engineering, College of Engineering, Qassim University, Unaizah, Saudi Arabia \\ ${ }^{3}$ Department of Mathematics, Riphah International University, Faisalabad, Pakistan \\ ${ }^{4}$ Department of Mathematics and Statistics, University of Agriculture, Faisalabad, Pakistan \\ ${ }^{5}$ School of Mathematics and Physics, Anhui Jianzhu University, Hefei 230601, China \\ Correspondence should be addressed to Haidar Ali; haidar3830@gmail.com
}

Received 18 September 2021; Accepted 9 October 2021; Published 1 November 2021

Academic Editor: Ljubisa Kocinac

Copyright (C) 2021 Wang Zhen et al. This is an open access article distributed under the Creative Commons Attribution License, which permits unrestricted use, distribution, and reproduction in any medium, provided the original work is properly cited.

\begin{abstract}
A molecular graph is used to represent a chemical molecule in chemical graph theory, which is a branch of graph theory. A graph is considered to be linked if there is at least one link between its vertices. A topological index is a number that describes a graph's topology. Cheminformatics is a relatively young discipline that brings together the field of sciences. Cheminformatics helps in establishing QSAR and QSPR models to find the characteristics of the chemical compound. We compute the first and second modified K-Banhatti indices, harmonic K-Banhatti index, symmetric division index, augmented Zagreb index, and inverse sum index and also provide the numerical results.
\end{abstract}

\section{Introduction}

Graph theory provides topological indices, which are a useful tool. Cheminformatics is a contemporary academic discipline that brings together chemistry, mathematics, and information science. It investigates the connections between quantitative structure-activity relationship (QSAR) and quantitative structure-property relationship (QSPR), which are used to predict biological activities and chemical compound characteristics.

The silicate structures [1] formed from the $\mathrm{POH}$ network, TP network, and hex $\mathrm{POH}$ network [2] are discussed in this article.

The following is the procedure for making $\mathrm{POH}$ networks .

Step 1: consider a $m$-dimensional silicate network.

Step 2: connect new vertices in the centre of each triangular face to existing vertices in the adjacent triangular face.
Step 3: all of the new centre vertices in the same silicate cell must be connected.

Step 4: for the $m$ dimension, the resultant graph is known as the planar octahedron network as shown in Figure 1. Remove all silicon vertices from the graph. The triangle prism network as shown in Figure 2 and the hex $\mathrm{POH}$ network as shown in Figure 3 are also possible.

Let $\psi$ represent a graph. Then, modified first and second $\mathrm{K}$-Banhatti indices [3] can be defined as

$$
\begin{aligned}
& \operatorname{MK}_{1} \mathrm{~B}(\psi)=\sum_{a b \in E(\psi)}\left(\frac{1}{\left(d_{a}+d_{b}\right)}\right), \\
& \mathrm{MK}_{2} \mathrm{~B}(\psi)=\sum_{a b \in E(\psi)}\left(\frac{1}{\left(d_{a} \times d_{b}\right)}\right) .
\end{aligned}
$$

Harmonic K-Banhatti index [4] of a graph $\psi$ is defined as 


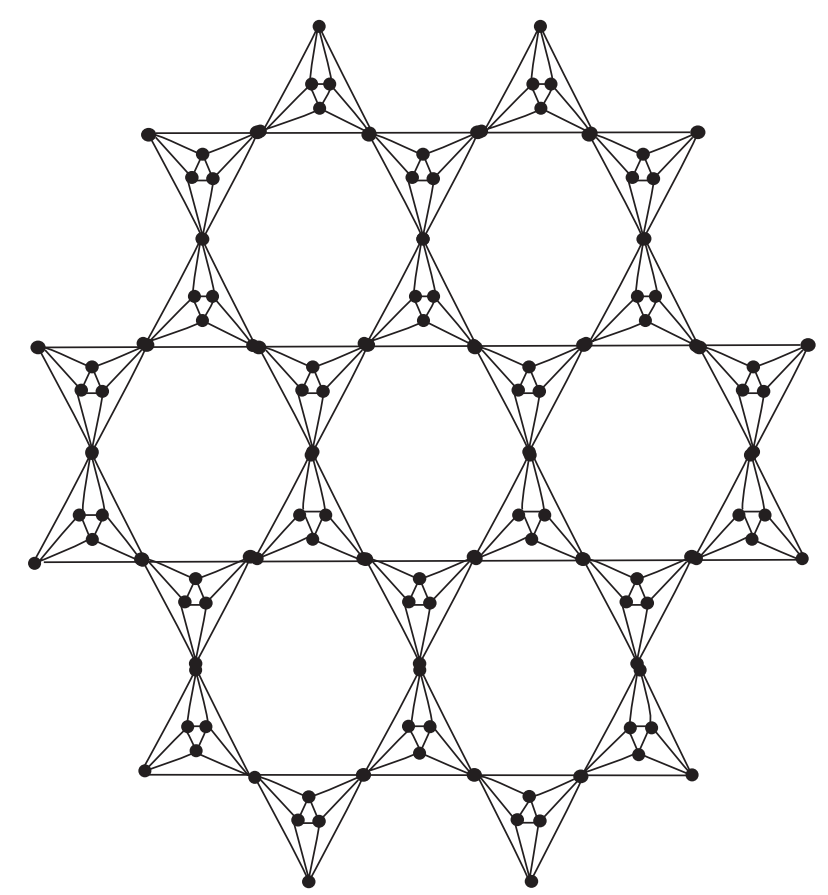

FIgURE 1: Planar octahedral network POH(2).

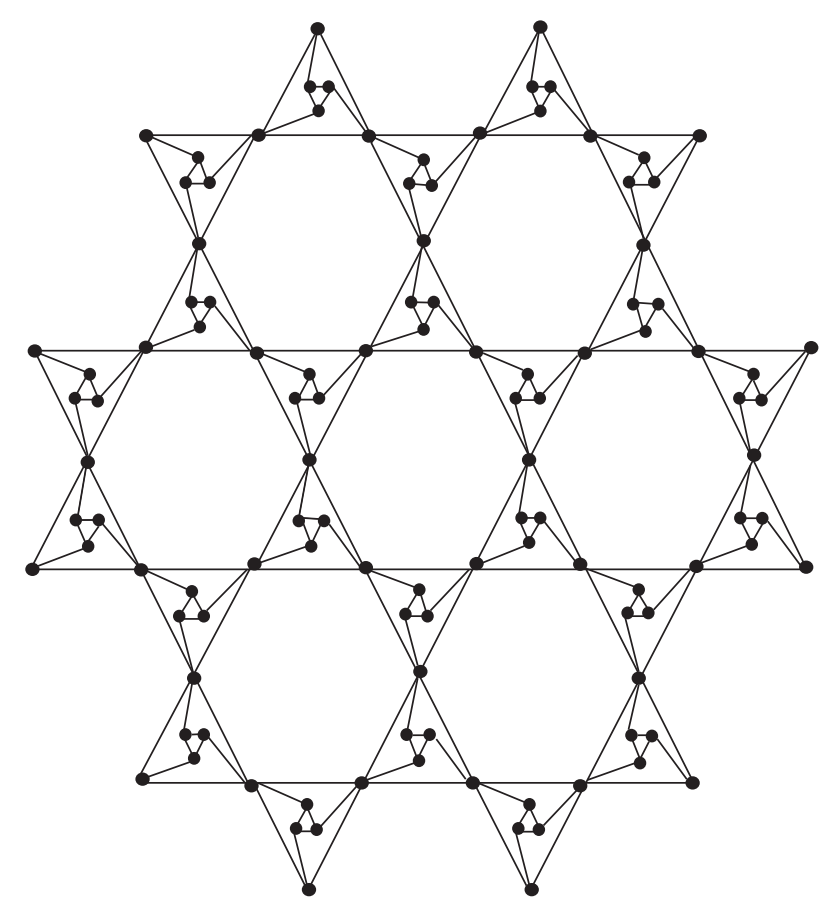

FIgURE 2: Triangular prism network TP(2).

$$
\operatorname{HKB}(\psi)=\sum_{a b \in E(\psi)}\left(\frac{2}{\left(d_{a}+d_{b}\right)}\right)
$$

Symmetric division index of a graph [5] is defined as

$$
\operatorname{SD}(\psi)=\sum_{a b \in E(\psi)}\left(\frac{d_{a}}{d_{b}}+\frac{d_{b}}{d_{a}}\right) .
$$

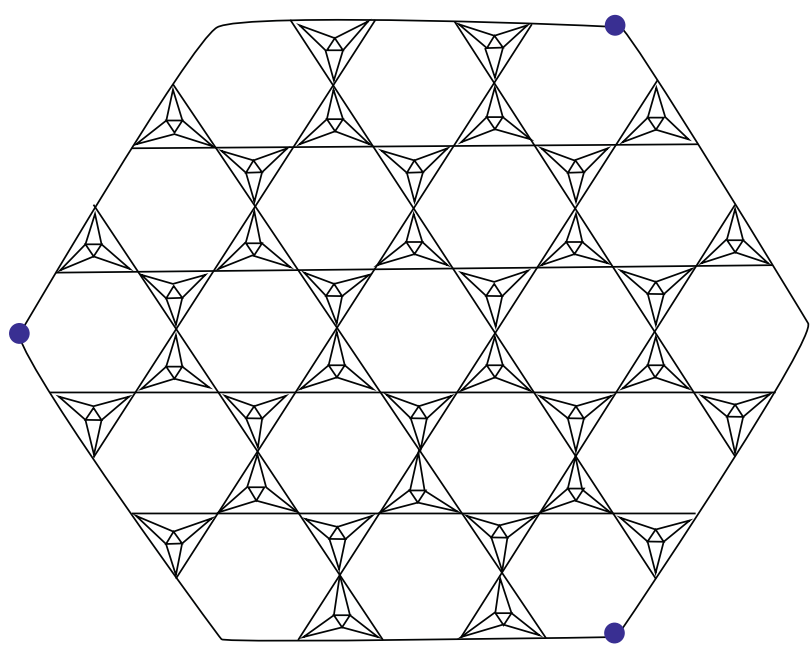

Figure 3: Hexagonal planar octahedral network $\mathrm{HPOH}(2)$.

Augmented Zagreb index of a graph $\psi[4]$ is defined as

$$
\operatorname{AG}(\psi)=\sum_{a b \in E(\psi)}\left(\frac{d_{a} \times d_{b}}{d_{a}+d_{b}-2}\right)^{3} \text {. }
$$

Inverse sum index of a graph $\psi$ is defined as

$$
I(\psi)=\sum_{a b \in E(\psi)}\left(\frac{d_{a} \times d_{b}}{d_{a}+d_{b}}\right) .
$$

\section{Main Results}

We research different indices on different kinds of planar octahedron networks. Nowadays, extensive research studies are being conducted in the field of chemical graph theory for further studying topological indices of various graphs [6-13]. For the basic notations and definitions, see $[14,15]$.

2.1. Results for Planar Octahedron Network $\mathrm{POH}(\mathrm{m})$. The planar octahedron network is the resulting graph for the $m$ dimension. All silicon vertices should be removed from the scene. There are also the triangular prism network and the hex POH network. Now, we calculate several key indices for the $\mathrm{POH}$ network in the following theorems.

Theorem 1. Consider the planar octahedral network $P O H$ $(m)$; then, its first and second modified $K$-Banhatti indices are equal to

$$
\begin{gathered}
\mathrm{MK}_{1} \mathrm{~B}\left(\psi_{1}\right)=\frac{51}{8} m^{2}+\frac{3}{4} m, \\
\mathrm{MK}_{2} \mathrm{~B}\left(\psi_{1}\right)=\frac{81}{32} m^{2}+\frac{9}{16} m .
\end{gathered}
$$

Proof. Let $\psi_{1} \cong \mathrm{POH}(m)$. From equation (1), we have 


$$
\mathrm{MK}_{1} \mathrm{~B}(\psi)=\sum_{a b \in E(\psi)}\left(\frac{1}{\left(d_{a}+d_{b}\right)}\right) .
$$

Using Table 1, we have
(8)

\begin{tabular}{lc}
\hline$\left(d_{a}, d_{b}\right)$ & Number of edges \\
\hline$E_{1}=(4,4)$ & $18 m^{2}+12 m$ \\
$E_{2}=(4,8)$ & $36 m^{2}-48 m+12$ \\
$E_{3}=(8,8)$ & $18 m^{2}-36 m+18$ \\
\hline
\end{tabular}

$$
\begin{aligned}
\mathrm{MK}_{1} \mathrm{~B}\left(\psi_{1}\right) & =\frac{1}{4+4}\left|E_{1}(\mathrm{POH}(m))\right|+\frac{1}{4+8}\left|E_{2}(\mathrm{POH}(m))\right|+\frac{1}{8+8}\left|E_{3}(\mathrm{POH}(m))\right| \\
& =\frac{1}{8}\left|E_{1}(\mathrm{POH}(m))\right|+\frac{1}{12}\left|E_{2}(\mathrm{POH}(m))\right|+\frac{1}{16}\left|E_{3}(\mathrm{POH}(m))\right| \\
& =\frac{1}{8}\left(18 m^{2}+12 m\right)+\frac{1}{12}\left(36 m^{2}-48 m+12\right)+\frac{1}{16}\left(18 m^{2}-36 m+18\right) .
\end{aligned}
$$

We get the following value after calculations:

$$
\mathrm{MK}_{1} \mathrm{~B}(\psi)=\sum_{a b \in E(\psi)}\left(\frac{1}{\left(d_{a}+d_{b}\right)}\right) .
$$

$$
\Rightarrow \mathrm{MK}_{1} \mathrm{~B}\left(\psi_{1}\right)=\frac{51}{8} m^{2}+\frac{3}{4} m .
$$

Using Table 1, we have

Let $\psi_{1} \cong \mathrm{POH}(m)$. From equation (2), we have

$$
\begin{aligned}
\mathrm{MK}_{2} \mathrm{~B}\left(\psi_{1}\right) & =\frac{1}{4 \times 4}\left|E_{1}(\mathrm{POH}(m))\right|+\frac{1}{4 \times 8}\left|E_{2}(\mathrm{POH}(m))\right|+\frac{1}{8 \times 8}\left|E_{3}(\mathrm{POH}(m))\right| \\
& =\frac{1}{16}\left|E_{1}(\mathrm{POH}(m))\right|+\frac{1}{32}\left|E_{2}(\mathrm{POH}(m))\right|+\frac{1}{64}\left|E_{3}(\mathrm{POH}(m))\right| \\
& =\frac{1}{16}\left(18 m^{2}+12 m\right)+\frac{1}{32}\left(36 m^{2}-48 m+12\right)+\frac{1}{64}\left(18 m^{2}-36 m+18\right) .
\end{aligned}
$$

We get the following value after calculations:

$$
\Rightarrow \mathrm{MK}_{2} \mathrm{~B}\left(\psi_{1}\right)=\frac{81}{32} m^{2}+\frac{9}{16} m \text {. }
$$

Proof. Let $\varphi_{1} \cong \mathrm{POH}(m)$ network; from equation (3),

$$
\operatorname{HKB}(\psi)=\sum_{a b \in E(\psi)} \frac{2}{\left(d_{a}+d_{b}\right)} .
$$

Theorem 2. The harmonic K-Banhatti and symmetric division indices are equal in the $\mathrm{POH}(\mathrm{m})$ network.

$$
\begin{aligned}
\operatorname{HKB}(\psi) & =\frac{51}{4} m^{2}+\frac{3}{2} m, \\
\operatorname{SD}(\psi) & =162 m^{2} .
\end{aligned}
$$

Using Table 1, we have

$$
\begin{aligned}
\operatorname{HKB}\left(\psi_{1}\right) & =\frac{2}{4+4}\left|E_{1}(\mathrm{POH}(m))\right|+\frac{2}{4+8}\left|E_{2}(\mathrm{POH}(m))\right|+\frac{2}{8+8}\left|E_{3}(\mathrm{POH}(m))\right| \\
& =\frac{2}{8}\left|E_{1}(\mathrm{POH}(m))\right|+\frac{2}{12}\left|E_{2}(\mathrm{POH}(m))\right|+\frac{2}{16}\left|E_{3}(\mathrm{POH}(m))\right| \\
& =\frac{1}{4}\left(18 m^{2}+12 m\right)+\frac{1}{6}\left(36 m^{2}-48 m+12\right)+\frac{1}{8}\left(18 m^{2}-36 m+18\right) .
\end{aligned}
$$

We get the following value after calculations:

$$
\Rightarrow \operatorname{HKB}\left(\psi_{1}\right)=\frac{51}{4} m^{2}+\frac{3}{2} m .
$$


For the symmetric division index of a graph using equation (3),

$$
\operatorname{SD}(\psi)=\sum_{a b \in E(\psi)}\left(\frac{d_{a}}{d_{b}}+\frac{d_{b}}{d_{a}}\right) .
$$

$$
\begin{aligned}
\mathrm{SD}\left(\psi_{1}\right) & =\left(\frac{4}{4}+\frac{4}{4}\right)\left|E_{1}(\mathrm{POH}(m))\right|+\left(\frac{4}{8}+\frac{8}{4}\right)\left|E_{2}(\mathrm{POH}(m))\right|+\left(\frac{8}{8}+\frac{8}{8}\right)\left|E_{3}(\mathrm{POH}(m))\right| \\
& =2\left|E_{1}(\mathrm{POH}(m))\right|+\left(\frac{1}{2}+\frac{2}{1}\right)\left|E_{2}(\mathrm{POH}(m))\right|+2\left|E_{3}(\mathrm{POH}(m))\right| \\
& =2\left(18 m^{2}+12 m\right)+\frac{5}{2}\left(36 m^{2}-48 m+12\right)+2\left(18 m^{2}-36 m+18\right) .
\end{aligned}
$$

After calculations,

$$
\Rightarrow \mathrm{SD}\left(\psi_{1}\right)=162 \mathrm{~m}^{2} \text {. }
$$

Theorem 3. Then, augmented Zagreb and inverse sum indices are equal to the $\mathrm{POH}$ network.

$$
\begin{gathered}
\operatorname{AG}\left(\psi_{1}\right)=\frac{416820224}{128625} m^{2}-\frac{2836480}{3087} m, \\
I\left(\psi_{1}\right) 204 m^{2}-24 m .
\end{gathered}
$$

Proof. Let $\varphi_{1} \cong \mathrm{POH}(m)$ network. For the augmented Zagreb index, using equation (5),

$$
\operatorname{AG}(\psi)=\sum_{a b \in E(\psi)}\left(\frac{d_{a} \times d_{b}}{d_{a}+d_{b}-2}\right)^{3} .
$$

Using Table 1, we have

$$
\begin{aligned}
\operatorname{AG}\left(\psi_{1}\right) & =\left(\frac{16}{6}\right)^{3}\left|E_{1}(\mathrm{POH}(m))\right|+\left(\frac{32}{10}\right)^{3}\left|E_{2}(\mathrm{POH}(m))\right|+\left(\frac{64}{14}\right)^{3}\left|E_{3}(\mathrm{POH}(m))\right| \\
& =\left(\frac{16}{6}\right)^{3}\left(18 m^{2}+12 m\right)+\left(\frac{32}{10}\right)^{3}\left(36 m^{2}-48 m+12\right)+\left(\frac{64}{14}\right)^{3}\left(18 m^{2}-36 m+18\right) .
\end{aligned}
$$

After some calculations, we get

$$
\Rightarrow \operatorname{AG}\left(\psi_{1}\right)=\frac{416820224}{128625} m^{2}-\frac{2836480}{3087} m \text {. }
$$

$$
I(\psi)=\sum_{a b \in E(\psi)}\left(\frac{d_{a} \times d_{b}}{d_{a}+d_{b}}\right) .
$$
have

For the inverse sum index and by using equation (6), we

Using Table 1, we have

$$
\begin{array}{r}
I\left(\psi_{1}\right)=\left(\frac{16}{8}\right)\left|E_{1}(\mathrm{POH}(m))\right|+\left(\frac{32}{12}\right)\left|E_{2}(\mathrm{POH}(m))\right|+\left(\frac{64}{16}\right)\left|E_{3}(\mathrm{POH}(m))\right| \\
=2\left(18 m^{2}+12 m\right)+\left(\frac{32}{12}\right)\left(36 m^{2}-48 m+12\right)+4\left(18 m^{2}-36 m+18\right) . \\
\Rightarrow I\left(\psi_{1}\right)=204 m^{2}-24 m .
\end{array}
$$

After calculations, 
2.2. Results for Triangular Prism Network TP $(m)$. In this part, we propose the theorem for the TP network.

Theorem 4. The first and second modified K-Banhatti indices are equal to the TP network:

$$
\begin{aligned}
& \mathrm{MK}_{1} \mathrm{~B}\left(\psi_{2}\right)=\frac{13}{2} m^{2}+\frac{2}{3} m, \\
& \mathrm{MK}_{2} \mathrm{~B}\left(\psi_{2}\right)=\frac{7}{2} m^{2}+\frac{2}{3} m .
\end{aligned}
$$

Proof. Let $\psi_{2} \cong \mathrm{TP}(m)$. From equation (1), we have
TABle 2: Edge partition of TP network.

\begin{tabular}{lc}
\hline$\left(d_{a}, d_{b}\right)$ & Number of edges \\
\hline$E_{1}=(3,3)$ & $18 m^{2}+6 m$ \\
$E_{2}=(3,6)$ & $18 m^{2}+6 m$ \\
$E_{3}=(6,6)$ & $18 m^{2}-36 m+18$ \\
\hline
\end{tabular}

$$
\mathrm{MK}_{1} \mathrm{~B}(\psi)=\sum_{a b \in E(\psi)}\left(\frac{1}{\left(d_{a}+d_{b}\right)}\right) .
$$

Using Table 2, we have

$$
\begin{aligned}
\mathrm{MK}_{1} \mathrm{~B}\left(\psi_{2}\right) & =\frac{1}{3+3}\left|E_{1}(\mathrm{TP}(m))\right|+\frac{1}{3+6}\left|E_{2}(\mathrm{TP}(m))\right|+\frac{1}{6+6}\left|E_{3}(\mathrm{TP}(m))\right| \\
& =\frac{1}{6}\left|E_{1}(\mathrm{TP}(m))\right|+\frac{1}{9}\left|E_{2}(\mathrm{TP}(m))\right|+\frac{1}{12}\left|E_{3}(\mathrm{TP}(m))\right| \\
& =\frac{1}{6}\left(18 m^{2}+6 m\right)+\frac{1}{9}\left(18 m^{2}+6 m\right)+\frac{1}{12}\left(18 m^{2}-36 m+18\right) .
\end{aligned}
$$

After some calculations, we get

$$
\Rightarrow \mathrm{MK}_{1} \mathrm{~B}\left(\psi_{2}\right)=\frac{13}{2} m^{2}+\frac{2}{3} m
$$

$$
\mathrm{MK}_{1} \mathrm{~B}(\psi)=\sum_{a b \in E(\psi)}\left(\frac{1}{\left(d_{a}+d_{b}\right)}\right) .
$$

Let $\psi_{2} \cong \mathrm{TP}(m)$. From equation (2), we have

$$
\begin{aligned}
\mathrm{MK}_{2} \mathrm{~B}\left(\psi_{2}\right) & =\frac{1}{3 \times 3}\left|E_{1}(\mathrm{TP}(m))\right|+\frac{1}{3 \times 6}\left|E_{2}(\mathrm{TP}(m))\right|+\frac{1}{6 \times 6}\left|E_{3}(\mathrm{TP}(m))\right| \\
& =\frac{1}{9}\left|E_{1}(\mathrm{TP}(m))\right|+\frac{1}{18}\left|E_{2}(\mathrm{TP}(m))\right|+\frac{1}{36}\left|E_{3}(\mathrm{TP}(m))\right| \\
& =\frac{1}{9}\left(18 m^{2}+6 m\right)+\frac{1}{18}\left(18 m^{2}+6 m\right)+\frac{1}{36}\left(18 m^{2}-36 m+18\right) .
\end{aligned}
$$

After calculations,

$$
\begin{aligned}
\operatorname{HKB}\left(\psi_{2}\right) & =13 m^{2}+\frac{4}{3} m \\
\operatorname{SD}\left(\psi_{2}\right) & =117 m^{2}+3 m
\end{aligned}
$$$$
\Rightarrow \mathrm{MK}_{2} \mathrm{~B}\left(\psi_{2}\right)=\frac{7}{2} m^{2}+\frac{2}{3} m .
$$

Theorem 5. In the POH $(m)$ network, the harmonic $K$-Banhatti and symmetric division indices are equal to
Proof. Let $\varphi_{2} \cong \mathrm{TP}(m)$ network, and by using equation (3), we have 


$$
\operatorname{HKB}(\psi)=\sum_{a b \in E(\psi)} \frac{2}{\left(d_{a}+d_{b}\right)}
$$

Using Table 2, we have

$$
\begin{aligned}
\operatorname{HKB}\left(\psi_{2}\right) & =\frac{2}{3+3}\left|E_{1}(\mathrm{TP}(m))\right|+\frac{2}{3+6}\left|E_{2}(\mathrm{TP}(m))\right|+\frac{2}{6+6}\left|E_{3}(\mathrm{TP}(m))\right| \\
& =\frac{2}{6}\left|E_{1}(\mathrm{TP}(m))\right|+\frac{2}{9}\left|E_{2}(\mathrm{TP}(m))\right|+\frac{2}{12}\left|E_{3}(\mathrm{TP}(m))\right| \\
& =\frac{1}{3}\left(18 m^{2}+6 m\right)+\frac{2}{9}\left(18 m^{2}+6 m\right)+\frac{1}{6}\left(18 m^{2}-36 m+18\right) .
\end{aligned}
$$

After calculations,

$$
\Rightarrow \operatorname{HKB}\left(\psi_{2}\right)=13 m^{2}+\frac{4}{3} m \text {. }
$$

$$
\operatorname{SD}(\psi)=\sum_{a b \in E(\psi)}\left(\frac{d_{a}}{d_{b}}+\frac{d_{b}}{d_{a}}\right) .
$$

For the symmetric division index of a graph using equation (3), we have

$$
\begin{aligned}
\operatorname{SD}\left(\psi_{2}\right) & =\left(\frac{3}{3}+\frac{3}{3}\right)\left|E_{1}(\operatorname{TP}(m))\right|+\left(\frac{3}{6}+\frac{6}{3}\right)\left|E_{2}(\operatorname{TP}(m))\right|+\left(\frac{6}{6}+\frac{6}{6}\right)\left|E_{3}(\operatorname{TP}(m))\right| \\
& =2\left|E_{1}(\operatorname{TP}(m))\right|+\left(\frac{1}{2}+2\right)\left|E_{2}(\operatorname{TP}(m))\right|+2\left|E_{3}(\operatorname{TP}(m))\right| \\
& =2\left(18 m^{2}+6 m\right)+\frac{5}{2}\left(18 m^{2}+6 m\right)+2\left(18 m^{2}-36 m+18\right)
\end{aligned}
$$

After some calculations, we get

$$
\Rightarrow \mathrm{SD}\left(\psi_{2}\right)=117 m^{2}+3 m
$$

Theorem 6. The augmented Zagreb and inverse sum indices are equal to the TP network.

$$
\begin{aligned}
\operatorname{AG}\left(\psi_{2}\right) & =\frac{1853423451}{1372000} m^{2}-\frac{534408759}{1372000} m \\
I\left(\psi_{2}\right) & =204 m^{2}-24 m
\end{aligned}
$$

Proof. Let $\varphi_{2} \cong \mathrm{POH}(m)$ network. Using equation (5) for the augmented Zagreb index,

$$
\operatorname{AG}(\psi)=\sum_{a b \in E(\psi)}\left(\frac{d_{a} \times d_{b}}{d_{a}+d_{b}-2}\right)^{3} .
$$

Using Table 2, we have

$$
\begin{aligned}
& \begin{aligned}
\operatorname{AG}\left(\psi_{2}\right) & =\left(\frac{9}{4}\right)^{3}\left|E_{1}(\mathrm{TP}(m))\right|+\left(\frac{18}{7}\right)^{3}\left|E_{2}(\mathrm{TP}(m))\right|+\left(\frac{36}{10}\right)^{3}\left|E_{3}(\mathrm{TP}(m))\right| \\
& =\left(\frac{9}{4}\right)^{3}\left(18 m^{2}+6 m\right)+\left(\frac{18}{7}\right)^{3}\left(18 m^{2}+6 m\right)+\left(\frac{36}{10}\right)^{3}\left(18 m^{2}-36 m+18\right) \\
& \Rightarrow \mathrm{AG}\left(\psi_{2}\right)=\frac{1853423451}{1372000} m^{2}-\frac{534408759}{1372000} m
\end{aligned}
\end{aligned}
$$

We get the following value after calculations: 

have

For the inverse sum index and by using equation (6), we Using Table 2, we have

$$
I(\psi)=\sum_{a b \in E(\psi)}\left(\frac{d_{a} \times d_{b}}{d_{a}+d_{b}}\right) .
$$

$$
\begin{aligned}
I\left(\psi_{2}\right) & =\left(\frac{9}{6}\right)\left|E_{1}(\operatorname{TP}(m))\right|+2\left|E_{2}(\operatorname{TP}(m))\right|+3\left|E_{3}(\operatorname{TP}(m))\right| \\
& =\left(\frac{9}{6}\right)\left(18 m^{2}+6 m\right)+2\left(18 m^{2}+6 m\right)+3\left(18 m^{2}-36 m+18\right) .
\end{aligned}
$$

After calculations,

$$
\begin{aligned}
& \mathrm{MK}_{1} \mathrm{~B}\left(\psi_{3}\right)=\frac{51}{8} m^{2}-4 m-\frac{13}{8}, \\
& \mathrm{MK}_{2} \mathrm{~B}\left(\psi_{3}\right)=\frac{81}{32} m^{2}-\frac{15}{16} m-\frac{39}{32} .
\end{aligned}
$$$$
\Rightarrow I\left(\psi_{2}\right)=117 m^{2}-15 m .
$$

2.3. Results for Hexagonal Planar Octahedron (HPOH) Network. In this part, we propose the theorem for the $\mathrm{HPOH}$ network.

Theorem 7. The first and second modified K-Banhatti indices are equal to the hex $\mathrm{POH}$ network:
Proof. Let $\psi_{3} \cong \mathrm{HPOH}(m)$. From equation (1), we have

$$
\mathrm{MK}_{1} \mathrm{~B}(\psi)=\sum_{a b \in E(\psi)}\left(\frac{1}{\left(d_{a}+d_{b}\right)}\right) .
$$

Using Table 3, we have

$$
\begin{aligned}
\mathrm{MK}_{1} \mathrm{~B}\left(\psi_{3}\right) & =\frac{1}{4+4}\left|E_{1}(\mathrm{HPOH}(m))\right|+\frac{1}{4+8}\left|E_{2}(\mathrm{HPOH}(m))\right|+\frac{1}{8+8}\left|E_{3}(\mathrm{HPOH}(m))\right| \\
& =\frac{1}{8}\left|E_{1}(\mathrm{HPOH}(m))\right|+\frac{1}{12}\left|E_{2}(\mathrm{HPOH}(m))\right|+\frac{1}{16}\left|E_{3}(\mathrm{HPOH}(m))\right| \\
& =\frac{1}{8}\left(18 m^{2}+18 m-30\right)+\frac{1}{12}\left(36 m^{2}-48 m+12\right)+\frac{1}{16}\left(18 m^{2}-36 m+18\right) .
\end{aligned}
$$

We get the following value after calculations:

$$
\Rightarrow \mathrm{MK}_{1} \mathrm{~B}\left(\psi_{3}\right)=\frac{51}{8} m^{2}-4 m-\frac{13}{8} .
$$

Let $\psi_{2} \cong \mathrm{HPOH}(m)$. From equation (2), we have

$$
\begin{aligned}
\operatorname{MK}_{2} \mathrm{~B}\left(\psi_{3}\right) & =\frac{1}{4 \times 4}\left|E_{1}(\mathrm{HPOH}(m))\right|+\frac{1}{4 \times 8}\left|E_{2}(\mathrm{HPOH}(m))\right|+\frac{1}{8 \times 8}\left|E_{3}(\operatorname{HPOH}(m))\right| \\
& =\frac{1}{16}\left|E_{1}(\operatorname{HPOH}(m))\right|+\frac{1}{32}\left|E_{2}(\operatorname{HPOH}(m))\right|+\frac{1}{64}\left|E_{3}(\mathrm{HPOH}(m))\right| \\
& =\frac{1}{16}\left(18 m^{2}+18 m-30\right)+\frac{1}{32}\left(36 m^{2}-48 m+12\right)+\frac{1}{64}\left(18 m^{2}-36 m+18\right) .
\end{aligned}
$$


TABLE 3: Edge partition.

\begin{tabular}{lc}
\hline$\left(d_{a}, d_{b}\right)$ & Number of edges \\
\hline$E_{1}=(4,4)$ & $18 m^{2}+18 m-30$ \\
$E_{2}=(4,8)$ & $36 m^{2}-48 m+12$ \\
$E_{3}=(8,8)$ & $18 m^{2}-36 m+18$ \\
\hline
\end{tabular}

We get the following value after calculations:

$$
\Rightarrow \mathrm{MK}_{2} \mathrm{~B}\left(\psi_{3}\right)=\frac{81}{32} m^{2}-\frac{15}{16} m-\frac{39}{32} \text {. }
$$

Theorem 8. Then, harmonic K-Banhatti and symmetric division indices are equal to the hex $\mathrm{POH}$ network:

$$
\begin{aligned}
\operatorname{HKB}\left(\psi_{3}\right) & =\frac{51}{4} m^{2}-8 m-\frac{13}{4}, \\
\operatorname{SD}\left(\psi_{3}\right) & =162 m^{2}-156 m+6 .
\end{aligned}
$$

Proof. Let $\varphi_{1} \cong \mathrm{HPOH}(m)$ network, and from equation (3),

$$
\operatorname{HKB}(\psi)=\sum_{a b \in E(\psi)} \frac{2}{\left(d_{a}+d_{b}\right)} .
$$

Using Table 3, we have

$$
\begin{aligned}
\operatorname{HKB}\left(\psi_{3}\right) & =\frac{2}{4+4}\left|E_{1}(\mathrm{HPOH}(m))\right|+\frac{2}{4+8}\left|E_{2}(\mathrm{HPOH}(m))\right|+\frac{2}{8+8}\left|E_{3}(\mathrm{HPOH}(m))\right| \\
& =\frac{2}{8}\left|E_{1}(\mathrm{HPOH}(m))\right|+\frac{2}{12}\left|E_{2}(\operatorname{HPOH}(m))\right|+\frac{2}{16}\left|E_{3}(\mathrm{HPOH}(m))\right| \\
& =\frac{1}{4}\left(18 m^{2}+18 m-30\right)+\frac{1}{6}\left(36 m^{2}-48 m+12\right)+\frac{1}{8}\left(18 m^{2}-36 m+18\right) .
\end{aligned}
$$

We get the following value after calculations:

$$
\Rightarrow \operatorname{HKB}\left(\psi_{3}\right)=\frac{51}{4} m^{2}-8 m-\frac{13}{4} \text {. }
$$

$$
\operatorname{SD}(\psi)=\sum_{a b \in E(\psi)}\left(\frac{d_{a}}{d_{b}}+\frac{d_{b}}{d_{a}}\right) .
$$

For the symmetric division index of a graph using equation (3), we have

$$
\begin{aligned}
\mathrm{SD}\left(\psi_{3}\right) & =\left(\frac{4}{4}+\frac{4}{4}\right)\left|E_{1}(\mathrm{HPOH}(m))\right|+\left(\frac{4}{8}+\frac{8}{4}\right)\left|E_{2}(\mathrm{HPOH}(m))\right|+\left(\frac{8}{8}+\frac{8}{8}\right)\left|E_{3}(\mathrm{HPOH}(m))\right| \\
& =2\left|E_{1}(\operatorname{HPOH}(m))\right|+\left(\frac{1}{2}+\frac{2}{1}\right)\left|E_{2}(\mathrm{HPOH}(m))\right|+2\left|E_{3}(\mathrm{HPOH}(m))\right| \\
& =2\left(18 m^{2}+18 m-30\right)+\frac{5}{2}\left(36 m^{2}-48 m+12\right)+2\left(18 m^{2}-36 m+18\right) .
\end{aligned}
$$

After calculations,

$$
\Rightarrow \mathrm{SD}\left(\psi_{3}\right)=162 m^{2}-156 m+6 .
$$

Theorem 9. The augmented Zagreb and inverse sum indices are equal to the hex $\mathrm{POH}$ network: 


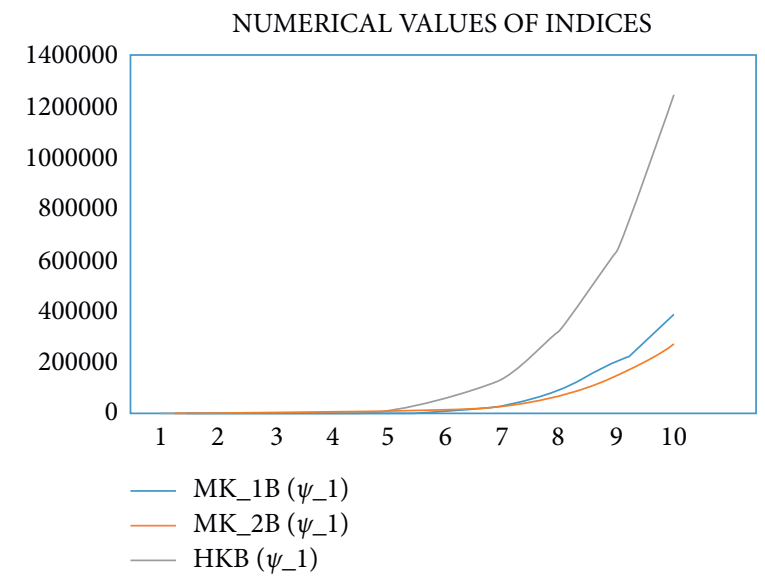

FIgURE 4: For POH 1.

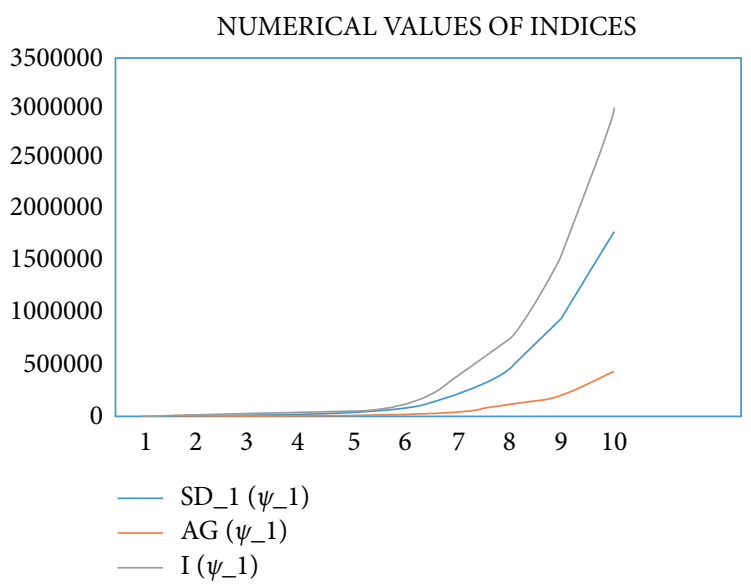

Figure 5: For POH 2.

$$
\begin{aligned}
\operatorname{AG}\left(\psi_{3}\right) & =\frac{416820224}{128625} m^{2}-\frac{600773632}{128625} m+\frac{595764224}{385875} \\
I\left(\psi_{3}\right) & =204 m^{2}-236 m+44
\end{aligned}
$$

Proof. Let $\varphi_{1} \cong \mathrm{HPOH}(m)$ network. Using equation (5) for the augmented Zagreb index,

$$
\operatorname{AG}(\psi)=\sum_{a b \in E(\psi)}\left(\frac{d_{a} \times d_{b}}{d_{a}+d_{b}-2}\right)^{3} .
$$

Using Table 3, we have

$$
\begin{aligned}
\operatorname{AG}\left(\psi_{3}\right) & =\left(\frac{16}{6}\right)^{3}\left|E_{1}(\operatorname{HPOH}(m))\right|+\left(\frac{32}{10}\right)^{3}\left|E_{2}(\operatorname{HPOH}(m))\right|+\left(\frac{64}{14}\right)^{3}\left|E_{3}(\operatorname{HPOH}(m))\right| \\
& =\left(\frac{16}{6}\right)^{3}\left(18 m^{2}+18 m-30\right)+\left(\frac{32}{10}\right)^{3}\left(36 m^{2}-48 m+12\right)+\left(\frac{64}{14}\right)^{3}\left(18 m^{2}-36 m+18\right) .
\end{aligned}
$$

We get the following value after calculations:

$\Rightarrow \mathrm{AG}\left(\psi_{3}\right)=\frac{416820224}{128625} m^{2}-\frac{600773632}{128625} m+\frac{595764224}{385875}$.
For the inverse sum index and by using equation (6), we have

$$
I(\psi)=\sum_{a b \in E(\psi)}\left(\frac{d_{a} \times d_{b}}{d_{a}+d_{b}}\right) .
$$




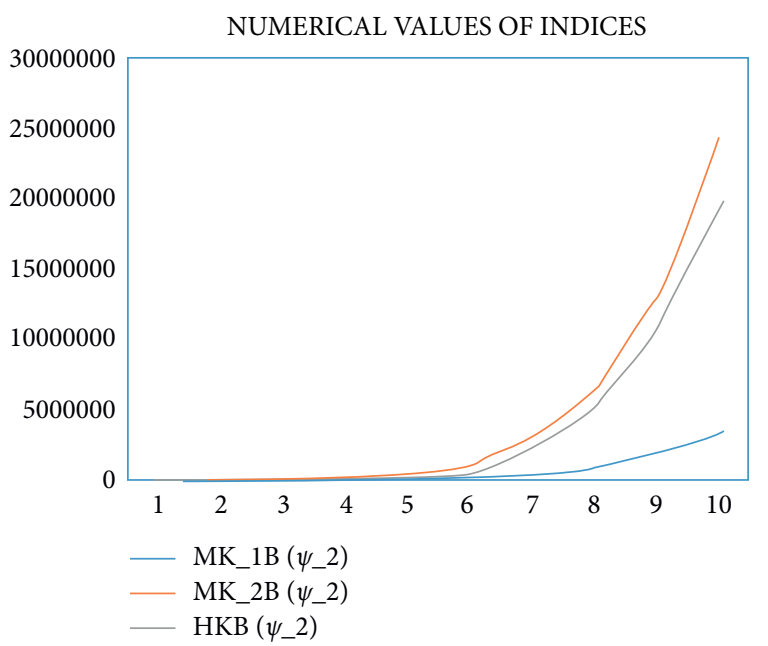

Figure 6: For TP 1.

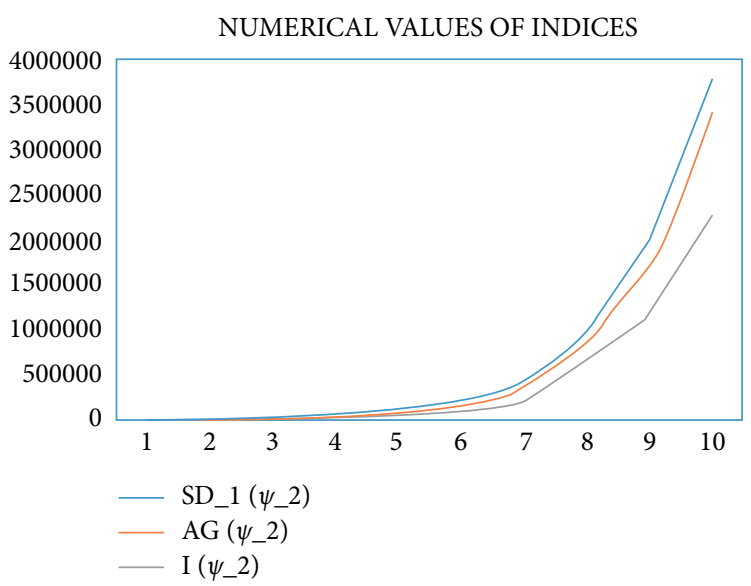

FIgURE 7: For TP 2.

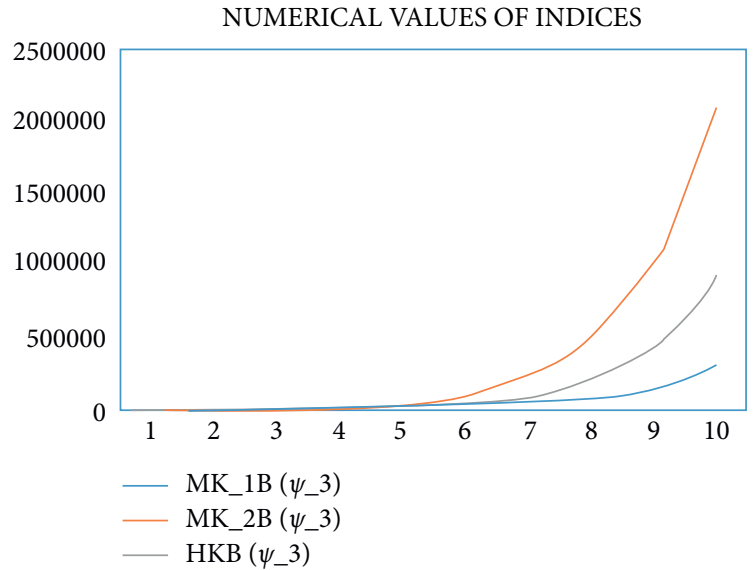

Figure 8: For hex POH 1. 


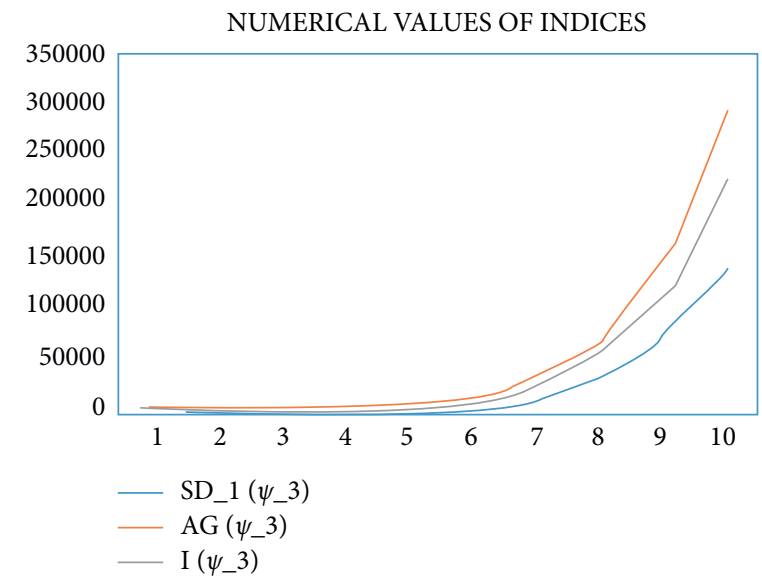

Figure 9: For hex POH 2.

Using Table 3, we have

$$
\begin{aligned}
I\left(\psi_{3}\right) & =\left(\frac{16}{8}\right)\left|E_{1}(\operatorname{HPOH}(m))\right|+\left(\frac{32}{12}\right)\left|E_{2}(\operatorname{HPOH}(m))\right|+\left(\frac{64}{16}\right)\left|E_{3}(\operatorname{HPOH}(m))\right| \\
& =2\left(18 m^{2}+18 m-30\right)+\left(\frac{32}{12}\right)\left(36 m^{2}-48 m+12\right)+4\left(18 m^{2}-36 m+18\right) .
\end{aligned}
$$

After calculations,

$$
\Rightarrow I\left(\psi_{3}\right)=204 m^{2}-236 m+44
$$

\section{Comparison of Indices through Graphs}

The comparison of the first and second K-Banhatti, harmonic K-Banhatti, symmetric division, augmented Zagreb, and inverse sum indices for the POH network, TP network, and HPOH network is conducted for different values. The comparison graphs are shown in Figures 4-9.

\section{Conclusion}

In this paper, first and the second K-Banhatti, harmonic K-Banhatti, symmetric division, augmented Zagreb, and inverse sum indices have been computed for the planar octahedron networks. From a chemical standpoint, these findings might be useful for computer scientists and chemists, who come across these networks. Additional multiplicative degree-based indices should be computed soon.

\section{Data Availability}

No data were used to support this study.

\section{Conflicts of Interest}

The authors declare that they have no conflicts of interest.

\section{Authors' Contributions}

Wang Zhen was responsible for software Parvez Ali contributed in collection of data. Haidar Ali contributed to original draft preparation. Ghulam Dustigeer was responsible for methodology. Jia-Bao Liu reviewed and edited the manuscript.

\section{Acknowledgments}

This research was supported by the Anhui Province University Discipline (Professional) Top Talent Academic Funding Project (grant no. gxbjZD2021091).

\section{References}

[1] P. D. Manuel and I. Rajasingh, "Minimum metric dimension of silicate networks," Ars Combinatoria, vol. 98, pp. 501-510, 2011.

[2] F. S. Raj and A. George, "Network embedding on planar octahedron networks," in Proceedings of the 2015 IEEE International Conference on Electrical, Computer and Communication Technologies (ICECCT), pp. 1-6, Coimbatore, India, March 2015.

[3] I. Gutman, B. Rusić, N. Trinajstić, and C. F. Wilcox Jr, "Graph theory and molecular orbitals. XII. Acyclic polyenes," The Journal of Chemical Physics, vol. 62, no. 9, pp. 3399-3405, 1975.

[4] V. R. Kulli, "Multiplicative hyper-Zagreb indices and coindices of graphs: computing these indices of some nanostructures," International Research Journal of Pure Algebra, vol. 6, no. 7, pp. 342-347, 2016. 
[5] J. B. Liu, Z. Jing, M. Jie, and C. Jinde, "The hosoya index of graphs formed by a fractal graph," Fractals, vol. 27, no. 8, Article ID 1950135, 2019.

[6] S. Akhtar and M. Imran, "On molecular topological properties of benzenoid structures," Canadian Journal of Chemistry, vol. 94, pp. 687-698, 2016.

[7] A. Q. Baig, M. Naeem, and W. Gao, "Revan and hyper-revan indices of octahedral and icosahedral networks," Applied Mathematics and Nonlinear Sciences, vol. 3, no. 1, pp. 33-40, 2018.

[8] W. Gao, A. Q. Baig, W. Khalid, and M. R. Farahani, "Molecular description of copper (II) oxide," Macedonian Journal of Chemistry and Chemical Engineering, vol. 36, pp. 93-99, 2017.

[9] M. Hu, H. Ali, M. A. Binyamin, B. Ali, J. B. Liu, and C. Fan, "On distance-based topological descriptors of chemical interconnection networks," Journal of Mathematics, vol. 2021, Article ID 5520619, 10 pages, 2021.

[10] F. Simonraj and A. George, "Embedding of poly honeycomb networks and the metric dimension of star of david network," International Journal on Applications of Graph Theory In wireless Ad Hoc Networks And sensor Networks, vol. 4, no. 4, pp. 11-28, 2012.

[11] G. H. Shirdel, H. Rezapour, and A. M. Sayadi, "The hyper Zagreb index of graph operations," Iranian Journal of Mathematical Chemistry, vol. 4, pp. 213-220, 2013.

[12] K. M. Smith, "On neighbourhood degree sequences of complex networks," Scientific Reports, vol. 9, no. 1, p. 8340, 2019.

[13] H. Wiener, "Structural determination of paraffin boiling points," Journal of the American Chemical Society, vol. 69, no. 1, pp. 17-20, 1947.

[14] M. V. Diudea, I. Gutman, and J. Lorentz, Molecular Topology, Babes-Bolyai University, Cluj-Napoca, Romania, 2001.

[15] N. Trinajstić, Chemical Graph Theory, CRC Press, Boca Raton, FL, USA, 1983. 